\title{
Serum albumin level as a potential marker for deciding chemotherapy or best supportive care in elderly, advanced non- small cell lung cancer patients with poor performance status
}

\author{
Satoshi Ikeda ${ }^{1,2^{*}}$, Hiroshige Yoshioka ${ }^{1}$, Satoshi $1 k e{ }^{1}$, Mitsunori Morita', Naoyuki Sone ${ }^{1}$, Takashi Niwa²,
} Akihiro Nishiyama', Toshihide Yokoyama', Akimasa Sekine ${ }^{2}$, Takashi Ogura ${ }^{2}$ and Tadashi Ishida'

\begin{abstract}
Background: There have been few data on the chemotherapy in elderly advanced non-small cell lung cancer (NSCLC) patients with poor performance status (PS), and usefulness of chemotherapy for such patients remains unclear. The objective of this study was to identify factors that predicted the survival benefit of chemotherapy.

Methods: All consecutive elderly patients ( $\geq 75$ years) with advanced NSCLC, Eastern Cooperative Oncology Group PS $\geq 2$, EGFR mutation wild type/unknown, and newly diagnosed from January 2009 to December 2012 at a tertiary hospital were retrospectively reviewed.

Results: We enrolled 59 patients, and 31 patients received at least one chemotherapy regimen (chemotherapy group). However, 28 patients received best supportive care (BSC) alone (BSC group). The proportion of PS 2 and serum albumin levels was significantly higher in the chemotherapy group than in the BSC group. In the chemotherapy group, log-rank testing did not show statistically significant differences in overall survival (OS) between the single-agent therapy group and carboplatin-based doublet therapy group; however, the OS of patients receiving chemotherapy for only 1 cycle (early termination) was significantly shorter than patients receiving chemotherapy for $\geq 2$ cycles. Hypoalbuminemia was not only a risk factor for the early termination of chemotherapy but also an independent prognostic factor in the chemotherapy group. A receiver operating characteristic curve analysis showed that the best cut-off value was $3.40 \mathrm{~g} / \mathrm{dL}$. In patients with serum albumin levels $\geq 3.40 \mathrm{~g} / \mathrm{dL}$, OS was significantly better in the chemotherapy group than in the BSC group $(p=0.0156)$, however, patients with serum albumin levels $<3.40 \mathrm{~g} / \mathrm{dL}$ exhibited poor prognosis regardless of the presence or absence of chemotherapy.
\end{abstract}

Conclusion: In the elderly NSCLC patients with poor PS, serum albumin levels may help identify certain patient populations more likely to receive a survival benefit of systemic chemotherapy.

Keywords: Non-small cell lung cancer, Elderly, Performance status, Albumin, Hypoalbuminemia

\footnotetext{
* Correspondence: isatoshi0112@gmail.com

'Department of Respiratory Medicine, Kurashiki Central Hospital, Miwa 1-1-1,

Kurashiki-city 710-8602, Japan

${ }^{2}$ Department of Respiratory Medicine, Kanagawa Cardiovascular and

Respiratory Center, Tomioka-Higashi 6-16-1, Kanazawa-ku, Yokohama-city

236-0051, Japan
}

\section{$\int$ Biomed Central}

(c) The Author(s). 2017 Open Access This article is distributed under the terms of the Creative Commons Attribution 4.0 International License (http://creativecommons.org/licenses/by/4.0/), which permits unrestricted use, distribution, and reproduction in any medium, provided you give appropriate credit to the original author(s) and the source, provide a link to the Creative Commons license, and indicate if changes were made. The Creative Commons Public Domain Dedication waiver (http://creativecommons.org/publicdomain/zero/1.0/) applies to the data made available in this article, unless otherwise stated. 


\section{Background}

Among patients newly diagnosed with non-small cell lung cancer (NSCLC) in developed countries, approximately $50 \%$ are $\geq 70$ years at the time of diagnosis [1], and 30\%$40 \%$ are with an Eastern Cooperative Oncology Group (ECOG) performance status (PS) $\geq 2$ [2]. Because older age and poor PS have often been related to the increased risk of toxicity associated with cytotoxic chemotherapy, such patients have often been excluded from clinical trials. To note, some randomized phase 3 trials of singleagent therapy have been conducted for elderly, advanced NSCLC patients. In the Elderly Lung Cancer Vinorelbine Italian Study (ELVIS), median overall survival (OS) was significantly better in the vinorelbine group than that in the best supportive care (BSC) group [3, 4]. The Multicenter Italian Lung Cancer in the Elderly Study (MILES) revealed that median OS in the gemcitabine group was almost equal to that in the vinorelbine group [5]. Subsequently, the WJTOG9904 trial [6] showed that patients treated with docetaxel had a significantly higher response rate and better progression-free survival (PFS) compared with patients taking vinorelbine. However, the difference in OS was not statistically significant, and severe neutropenia was more common with docetaxel. In addition, trials of platinum-based doublet therapy have also been conducted in elderly patients. In a French Intergroup Study (IFCT-0501), OS was significantly betterin the carboplatin plus weekly paclitaxel group than that in the single-agent therapy (gemcitabine or vinorelbine) group [7]. However, grade $\geq 3$ neutropenia and treatmentrelated death was more common with carboplatin plus weekly paclitaxel compared with single-agent therapy. Based on these trial results, single-agent therapy (docetaxel, gemcitabine, or vinorelbine) was recommended as first-line treatment for elderly, advanced NSCLC patients without known driver mutations, and carboplatin-based doublet therapy may be a viable option in patients deemed able to tolerate such therapy. However, little is known concerning chemotherapy in elderly, advanced NSCLC patients with poor PS, and the usefulness of chemotherapy for such patients remains unclear. Moreover, elderly patients who are enrolled in clinical trials represent a carefully selected subset. In clinical practice, elderly patients are a more heterogeneous population, with baseline organ dysfunctions and variable comorbidities, and the PS alone is not sufficient enough to account for the heterogeneity within elderly patients. It is critically important to identify patient populations that can receive a survival benefit of systemic chemotherapy in elderly patients with poor PS. In the present study, we retrospectively reviewed consecutive elderly patients $(\geq 75$ years of age) with advanced NSCLC and with poor PS (ECOG $P S \geq 2$ ) to identify factors that predict the survival benefit of cytotoxic chemotherapy.

\section{Methods}

\section{Patients and settings}

All consecutive patients enrolled were (1) pathologically or cytologically confirmed NSCLC; (2) at stage IIIB or IV according to the 7th edition TNM classification; (3) $\geq 75$ years of age; (4) with an Eastern Cooperative Oncology Group (ECOG) performance status (PS) $\geq 2$; (5) with an epidermal growth factor receptor mutation wild type or unknown status; and (6) newly diagnosed at the Kurashiki Central Hospital (Kurashiki city, Okayama, Japan) from January 2009 to December 2012. The exclusion criteria included clinical diagnosis of lung cancer without pathological or cytological confirmation. In patients with ECOG PS $\geq 3$, chemotherapy could be carried out only when the patient was diagnosed as treatable and tolerable for chemotherapy by the attending physician, and the patient and family were strongly hoping for the chemotherapy, even though they knew all the risks. This study has been carried out in accordance with the Declaration of Helsinki. The Ethics Committee of the Kurashiki Central Hospital approved the study protocol, and patient consent was waived because this was a retrospective study and anonymity was secured.

\section{Clinical and laboratory findings}

Clinical and laboratory data used in this study were retrieved from patient medical records and included age; gender; the ECOG PS; smoking status; comorbidities; tumor histology; cancer stage; major diameter of the primary site; metastatic organs (brain, bone, liver, and adrenal gland); laboratory data such as white blood cell, neutrophil, and lymphocyte counts as well as hemoglobin, albumin, lactate dehydrogenase, serum calcium, and Creactive protein levels; treatment status; progression free survival (PFS) of initial treatment; and OS. The OS was defined as the length of time from the date of diagnosis to death of any cause.

\section{Statistical analysis}

Categorical data are presented as numbers (percentages), whereas continuous data are presented as medians (interquartile ranges). Fisher's exact test was used to compare categorical data, and the Mann-Whitney U test was used to compare continuous data. Cumulative survival probabilities were estimated using the KaplanMeier method. The log-rank test was used to compare survival among patient groups. A multivariate analysis using a Cox proportional hazard model was performed to identify the factors associated with survival. A multivariate logistic regression analysis was performed to verify the risk factor for a categorical dependent variable. The factors with $p$-values $<0.05$ in univariate analysis were selected as candidate factors of multivariate analysis. A receiver operating characteristic (ROC) curve 
analysis was used to determine the optimal cut-off values for the risk factor; values with maximum joint sensitivity and specificity were selected. A $p$-value of $<0.05$ was considered statistically significant.

\section{Results}

\section{Baseline characteristics and prognoses in the study} population

In the present study, 59 patients were enrolled. Thirtyone patients received at least one chemotherapy regimen (chemotherapy group), whereas 28 patients received best supportive care (BSC) alone (BSC group). Patients' characteristics are summarized in Table 1 . The proportion of PS 2, lymphocyte count, and serum albumin level were significantly higher in the chemotherapy group than in the BSC group. No significant differences were observed regarding other clinical and laboratory data. A comparison of survival curves is shown in Fig. 1. The OS was better in the chemotherapy group than in the BSC group (median OS of 4.7 months and 3.1 months, $p=0.0119$ ).

\section{Treatment details and prognosis in the chemotherapy group}

Treatment details in the chemotherapy group are shown in Table 2. Twenty of the 31 patients $(64.5 \%)$ received singleagent therapy, whereas 11 of the 31 patients (35.5\%) received carboplatin-based doublet therapy. Patients who received carboplatin-based doublet therapy had higher response rates, and the median PFS values were better. No significant differences were observed in the disease control rate and median number of treatment cycles. An adverse event was the most common cause of cessation in patients receiving single-agent therapy, whereas, in patients receiving carboplatin-based doublet therapy, completion of 4-6 courses was the most common, followed by an adverse event. With regard to OS, log-rank testing did not show

Table 1 Baseline characteristics of the study population

\begin{tabular}{|c|c|c|c|}
\hline & Chemotherapy $(N=31)$ & Best supportive care $(N=28)$ & $p$-value \\
\hline Age & $78.0[76.5-80.0]$ & $80.5[77.0-84.3]$ & 0.118 \\
\hline Gender (male/female) & $4 / 27$ & $4 / 24$ & 1.00 \\
\hline ECOG Performance Status (2/3/4) & 18/12/1 & $8 / 15 / 5$ & 0.0350 \\
\hline Smoking history & $30(96.8 \%)$ & $22(78.6 \%)$ & 0.0870 \\
\hline Brinkman Index & 1100 [780-1550] & 800 [420-1395] & 0.113 \\
\hline \multicolumn{4}{|l|}{ Comorbidities } \\
\hline Emphysema (\%) & $26(83.9 \%)$ & $20(71.4 \%)$ & 0.348 \\
\hline Interstitial pneumonia (\%) & $1(3.2 \%)$ & $1(3.6 \%)$ & 1.00 \\
\hline Diabetes mellitus (\%) & $13(41.9 \%)$ & $1(3.6 \%)$ & 0.00100 \\
\hline Histology (Non-Squamous/Squamous) & $6 / 25$ & $6 / 22$ & 1.00 \\
\hline Staging (IIB/IV) & $5 / 26$ & $2 / 26$ & 0.428 \\
\hline Major diameter of the primary site & $39.5[27.5-65.0]$ & $52.0[40.5-74.0]$ & 0.0740 \\
\hline \multicolumn{4}{|l|}{ Metastatic organ } \\
\hline Brain (\%) & $6(19.4 \%)$ & $4(14.3 \%)$ & 0.734 \\
\hline Bone (\%) & $10(32.3 \%)$ & $6(21.4 \%)$ & 0.393 \\
\hline Liver (\%) & $3(9.7 \%)$ & $3(10.7 \%)$ & 1.00 \\
\hline Adrenal gland (\%) & $4(12.9 \%)$ & $1(3.6 \%)$ & 0.356 \\
\hline \multicolumn{4}{|l|}{ Laboratory data } \\
\hline White blood cell count & 7800 [6450-9700] & 7700 [6175-11,925] & 0.802 \\
\hline Neutrophil count & 5336 [4502-6684] & 5988 [4513-8929] & 0.362 \\
\hline Lymphocyte count & 1396 [1148-1721] & $1086[848-1432]$ & 0.0370 \\
\hline Hemoglobin & $12.4[11.0-14.0]$ & $12.0[11.5-13.2]$ & 0.885 \\
\hline Albumin & 3.60 [3.20-3.95] & $3.30[2.85-3.62]$ & 0.0460 \\
\hline Lactate dehydrogenase & 214 [188-247] & 247 [206-277] & 0.141 \\
\hline Calcium & $9.20[9.00-9.55]$ & $9.00[8.47-9.33]$ & 0.093 \\
\hline C-reactive protein & $1.81[0.66-3.92]$ & $3.20[0.57-7.59]$ & 0.391 \\
\hline
\end{tabular}

Categorical data are presented as numbers (percentages) whereas continuous data are presented as medians (interquartile ranges). Fisher's exact test was used to compare categorical data, and the Mann-Whitney $\mathrm{U}$ test was used to compare continuous data Abbreviations: ECOG Eastern Cooperative Oncology Group 


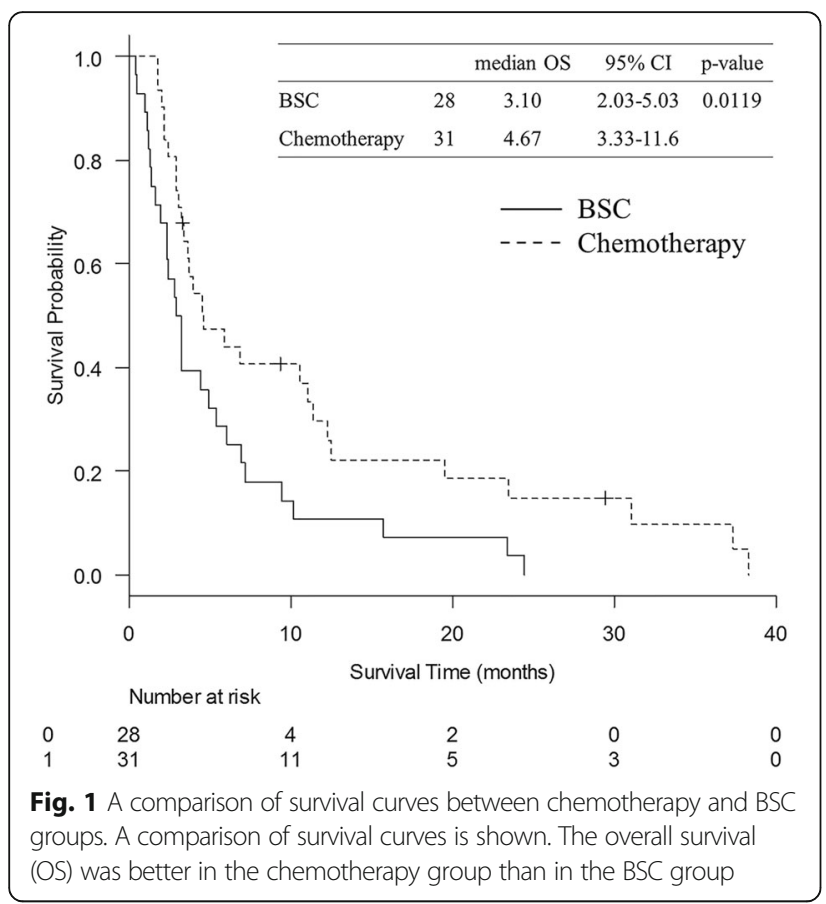

Table 2 Treatment details and prognoses of first-line chemotherapy

\begin{tabular}{|c|c|c|}
\hline & $\begin{array}{l}\text { Single-agent } \\
(N=20)\end{array}$ & $\begin{array}{l}\text { Platinum doublet } \\
(N=11)\end{array}$ \\
\hline \multicolumn{3}{|l|}{ Regimen } \\
\hline Gemcitabine & $8(40.0 \%)$ & 0 \\
\hline Vinorelbine & $6(30.0 \%)$ & 0 \\
\hline Docetaxel & $5(25.0 \%)$ & 0 \\
\hline Pemetrexed & $1(5.0 \%)$ & 0 \\
\hline Carboplatin + weekly paclitaxel & 0 & $9(81.8 \%)$ \\
\hline Carboplatin + gemcitabine & 0 & $1(9.1 \%)$ \\
\hline Carboplatin + S-1 & 0 & $1(9.1 \%)$ \\
\hline Response rate (\%) & 0 & $45.4 \%$ \\
\hline Disease control rate (\%) & $55.0 \%$ & $54.5 \%$ \\
\hline Progression free survival (month) & $\begin{array}{l}2.87 \\
{[0.60-7.27]}\end{array}$ & $\begin{array}{l}5.43 \\
{[1.58-8.07]}\end{array}$ \\
\hline Number of treatment cycles & $\begin{array}{l}2.00 \\
{[1.00-2.25]}\end{array}$ & $\begin{array}{l}3.00 \\
{[1.00-4.00]}\end{array}$ \\
\hline Early termination (only 1 cycle) (\%) & $7(35.0 \%)$ & $4(36.4 \%)$ \\
\hline \multicolumn{3}{|l|}{ Cause of cessation } \\
\hline Adverse event & $11(55.0 \%)$ & $4(36.4 \%)$ \\
\hline Deterioration of physical condition & $5(25.0 \%)$ & 0 \\
\hline Completion of 4-6 cycles & 0 & $5(45.5 \%)$ \\
\hline Progressive disease & $3(15.0 \%)$ & $1(9.1 \%)$ \\
\hline Patient's request & 1 (5.0\%) & 1 (9.1\%) \\
\hline
\end{tabular}

Categorical data are presented as numbers (percentages) whereas continuous data are presented as medians (interquartile ranges) statistically significant differences between the single-agent therapy and carboplatin-based doublet therapy groups (median OS of 3.80 months and 7.00 months, $p=0.773$ ) (Fig. 2a). On the other hand, the OS of patients receiving chemotherapy for only 1 cycle was significantly shorter than patients receiving chemotherapy for $\geq 2$ cycles (median OS of 3.0 months and 11.6 months, $p=0.0000241$ ) (Fig. 2b).

Risk factors for the early termination of chemotherapy Eleven patients received chemotherapy for only 1 cycle (early termination group), whereas 20 patients received chemotherapy for $\geq 2$ cycles (continuous treatment group). When comparing the clinical and laboratory data between two groups (Additional file 1 Table S1), the incidence of bone metastasis was higher and serum albumin levels were lower in the early termination group than in the continuous treatment group. No significant differences were observed for any other clinical and laboratory data.

A logistic regression analysis was performed to verify the risk factor for the early termination of chemotherapy (Table 3). In univariate analysis, serum albumin level and the existence of bone metastasis, all with $p$-values $<0.05$, were selected as candidate risk factors. A multivariate analysis showed that low serum albumin level and the existence of bone metastasis were significantly associated with the early termination of chemotherapy ( $p=0.0493$ and 0.0174 , respectively).

\section{The prognostic factors in the chemotherapy group}

An analysis using a Cox proportional hazard model was performed to verify the prognostic factor associated with survival in the chemotherapy group (Table 4). In univariate analysis, serum albumin level, number of cycles, the existence of bone metastasis, and the existence of adrenal gland metastasis, all with $p$-values $<0.05$, were selected as candidate factors. A multivariate analysis identified the serum albumin level as an independent factor associated with survival [hazard ratio: 0.174; $95 \%$ confidence interval (CI): 0.0610-0.495; $p=0.00104]$.

\section{Best cut off value for the serum albumin level}

To determine the cut-off values of serum albumin level for the "early termination of chemotherapy," an ROC curve analysis was performed. The area under the curve for the serum albumin level was 0.752 (95\% CI: 0.570 0.934 ) and the cut-off value for which sensitivity + specificity was maximal was $3.40 \mathrm{~g} / \mathrm{dL}(81.8 \%$ sensitivity and $70.0 \%$ specificity).

In addition, we performed a ROC curve analysis to determine the cut-off values of serum albumin level for "death within 3 months" in the chemotherapy group, which was based on the median OS of 3.1 months in the BSC group in the present study. The area under the curve for the serum albumin level was 0.739 (95\% CI: 


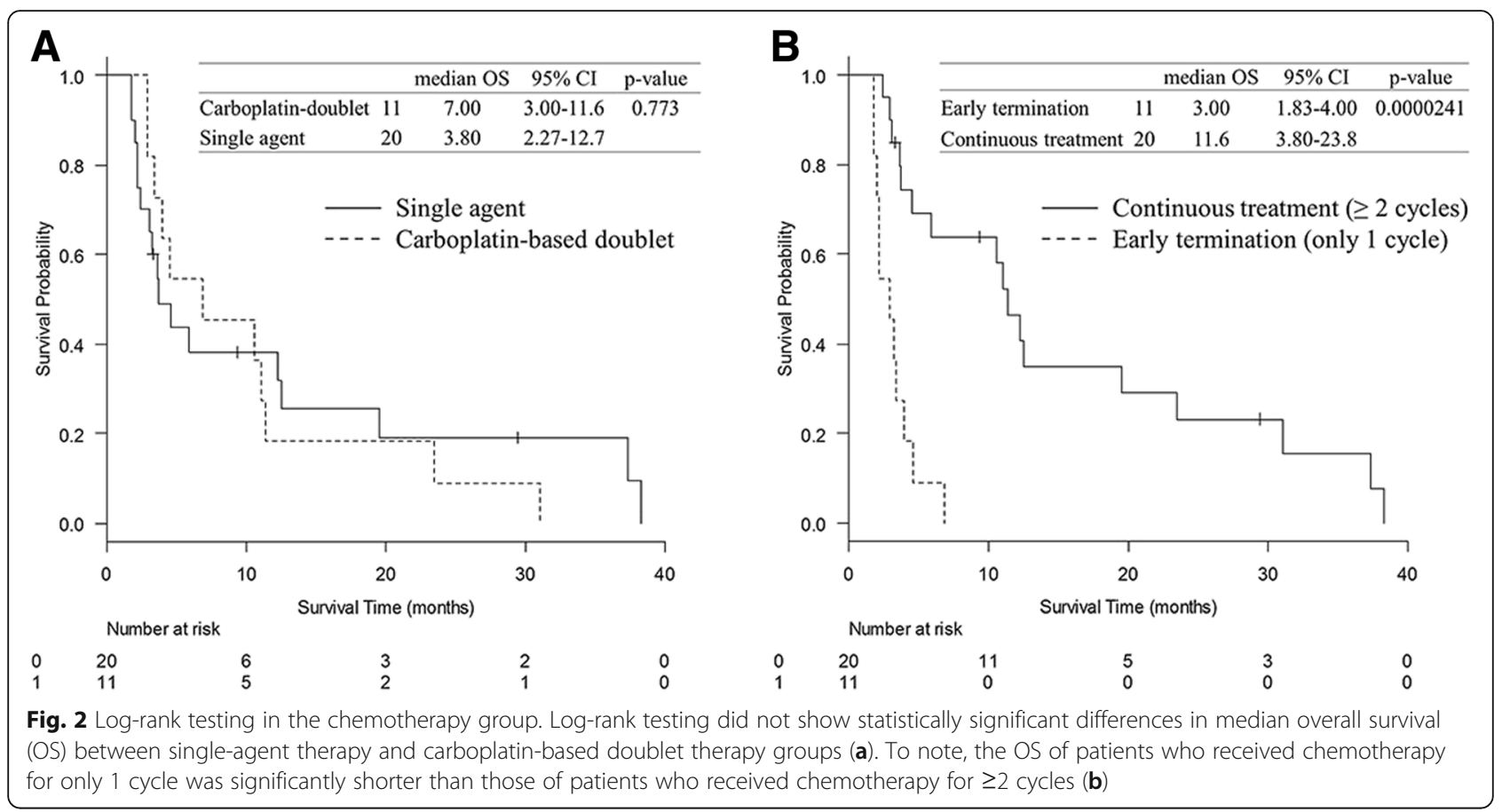

0.531-0.947) and the cut-off value for which sensitivity + specificity was maximal was also $3.40 \mathrm{~g} / \mathrm{dL}$ (87.5\% sensitivity and $65.2 \%$ specificity).

\section{Comparison of survival curves based on serum albumin levels}

We compared the survival curves between the BSC and chemotherapy groups based on the serum albumin level. For patients with serum albumin levels $\geq 3.40 \mathrm{~g} / \mathrm{dL}$, OS was significantly better in the chemotherapy group than that in the BSC group (respective median OS of 12.7 months and 3.9 months, $p=0.0156$ ) (Fig. 3a). In patients with serum albumin levels $<3.40 \mathrm{~g} / \mathrm{dL}$, the OS did not differ between the chemotherapy and BSC groups (respective median OS of 3.3 months and 2. 7 months, $p=0.620$ ) (Fig. 3b).

\section{Discussion}

The present study demonstrated the following three important clinical observations. First, the OS of the chemotherapy group was better than that of the BSC group in elderly patients with poor PS. Second, the number of treatment cycles had a larger impact on the survival benefit of chemotherapy than the decision/selection of either single-agent therapy or carboplatin-doublet therapy. Third, hypoalbuminemia was not only the risk factor for early termination of chemotherapy, but also the independent prognostic factor in the chemotherapy group.

The clinician-estimated PS is the most common method to evaluate physiologic reserve and functional status in NSCLC patients, and it is used to assess a patient's tolerability against chemotherapy. In previous clinical trials conducted for elderly, advanced NSCLC patients, such as the ELVIS and IFCT-0501 trials [3, 4, 7], 20-30\% of patients had a PS of 2, whereas almost no data were available for patients with $P S \geq 3$. Given this, there is a general consensus that elderly patients with PS 2 who wish to receive treatment should be offered chemotherapy, and elderly patients with $\mathrm{PS} \geq 3$ should receive supportive care aimed at maintaining quality of life [8]. In the present study, because of the differences in the baseline characteristics between the chemotherapy and BSC groups, it cannot be simply considered that chemotherapy prolonged OS in elderly patients with poor PS. However, meta-analysis of the clinical trials comparing chemotherapy and BSC for advanced NSCLC demonstrated that chemotherapy improves OS even in patients with poor PS [9]. Moreover, when comparing patients with PS 2 and PS $\geq 3$ in the chemotherapy group of the present study, there were no significant differences in the median number of initial treatment cycles (2 cycles each), disease control rates of the initial treatment (64.7\% in PS 2 and $66.7 \%$ in PS $\geq 3$ ), and median OS (6.50 months in PS 2 and 4.00 months in PS $\geq$ $3, p=0.987)$, regardless of the chemotherapy regimen. These results indicated that PS tends to be insufficient for assessing tolerability against chemotherapy and prognosis in elderly patients. Thus, there would be a certain population within elderly patients with poor PS to benefit via survival due to systemic chemotherapy. Especially in elderly patients, PS easily fluctuates based on various factors, such as pain caused by 
Table 3 Logistic regression analysis verifying the risk factors for early termination of chemotherapy $(N=31)$

\begin{tabular}{|c|c|c|c|}
\hline & $\begin{array}{l}\text { Odds } \\
\text { ratio }\end{array}$ & $\begin{array}{l}95 \% \text { confidence } \\
\text { interval }\end{array}$ & $p$-value \\
\hline \multicolumn{4}{|l|}{ Univariate analysis } \\
\hline Age & 0.93 & $0.722-1.20$ & 0.575 \\
\hline ECOG Performance status = 2 & 0.449 & $0.100-2.01$ & 0.295 \\
\hline Brinkman Index & 1.00 & $0.999-1.00$ & 0.655 \\
\hline Emphysema & 0.794 & $0.112-5.66$ & 0.818 \\
\hline Diabetes melitus & 2.23 & $0.497-10.0$ & 0.295 \\
\hline Squamous cell carcinoma & 0.889 & $0.135-5.85$ & 0.902 \\
\hline $\begin{array}{l}\text { Major diameter of the } \\
\text { primary site }\end{array}$ & 1.01 & $0.973-1.04$ & 0.672 \\
\hline Brain metastasis & 2.12 & $0.349-13.0$ & 0.414 \\
\hline Bone metastasis & 9.92 & $1.75-56.3$ & 0.00961 \\
\hline Liver metastasis & 0.900 & $0.0723-11.2$ & 0.935 \\
\hline Adrenal gland metastasis & 7.12 & $0.640-79.3$ & 0.110 \\
\hline $\begin{array}{l}\text { Carboplatin-based doublet } \\
\text { therapy }\end{array}$ & 1.06 & $0.229-4.92$ & 0.939 \\
\hline Lymphocyte count & 1 & $0.999-1.00$ & 0.866 \\
\hline Hemoglobin & 0.637 & $0.392-1.04$ & 0.0691 \\
\hline Albumin & 0.117 & $0.0168-0.811$ & 0.0299 \\
\hline Lactate dehydrogenase & 1.01 & $0.999-1.02$ & 0.0979 \\
\hline Calcium & 1.19 & $0.632-2.24$ & 0.59 \\
\hline C-reactive protein & 1.15 & $0.897-1.48$ & 0.267 \\
\hline \multicolumn{4}{|l|}{ Multivariate analysis } \\
\hline Bone metastasis & 10.9 & $1.52-77.9$ & 0.0174 \\
\hline Albumin & 0.0886 & $0.00791-0.992$ & 0.0493 \\
\hline
\end{tabular}

In the univariate analysis, serum albumin level and the existence of bone metastasis, all with $p$-values $<0.05$, were selected as candidate risk factors. A multivariate analysis showed that the association between serum albumin level and the existence of bone metastasis with early termination of chemotherapy were statistically significant

Abbreviations: ECOG Eastern Cooperative Oncology Group

cancer; thus, treatment decision-making should not be made based on temporal PS alone.

When performing chemotherapy, the optimal regimen for elderly patients with poor PS remains controversial. Carboplatin-based doublet therapy is clearly superior to single-agent therapy regarding antitumor effect, but it results in higher toxicity. In the present study, the response rate was higher and PFS was better in the carboplatin-doublet patient group than the response rate and PFS in the single-agent group (Table 2). However, there were no significant differences observed in the OS between the two groups (Fig. 2a). In previous randomized control trials designed for elderly populations tasked to compare non-platinum single agent and platinum-doublet therapies, only the IFCT-0501 trial showed the survival benefit of carboplatin plus weekly paclitaxel, even in patients with PS 2 [7], whereas other
Table 4 Analysis using a Cox proportional hazard model to verify the prognostic factor associated with survival in the chemotherapy group $(N=31)$

\begin{tabular}{|c|c|c|c|}
\hline & $\begin{array}{l}\text { Hazard } \\
\text { ratio }\end{array}$ & $\begin{array}{l}95 \% \text { confidence } \\
\text { interval }\end{array}$ & $p$-value \\
\hline \multicolumn{4}{|l|}{ Univariate analysis } \\
\hline Age & 0.995 & $0.884-1.12$ & 0.929 \\
\hline ECOG Performance status $=2$ & 0.994 & $0.453-2.18$ & 0.987 \\
\hline Brinkman Index & 0.999 & $0.998-1.00$ & 0.104 \\
\hline Emphysema & 0.649 & $0.241-1.74$ & 0.391 \\
\hline Diabetes mellitus & 0.990 & $0.448-2.189$ & 0.980 \\
\hline Squamous cell carcinoma & 1.15 & $0.420-3.12$ & 0.792 \\
\hline $\begin{array}{l}\text { Major diameter of the } \\
\text { primary site }\end{array}$ & 1.02 & $0.997-1.03$ & 0.102 \\
\hline Brain metastasis & 2.82 & $0.986-8.04$ & 0.0533 \\
\hline Bone metastasis & 3.07 & $1.24-7.57$ & 0.0150 \\
\hline Liver metastasis & 1.29 & $0.294-5.65$ & 0.736 \\
\hline Adrenal gland metastasis & 4.77 & $1.21-18.8$ & 0.0253 \\
\hline $\begin{array}{l}\text { Carboplatin-based doublet } \\
\text { therapy }\end{array}$ & 1.12 & $0.513-2.46$ & 0.773 \\
\hline $\begin{array}{l}\text { Number of treatment } \\
\text { cycles }\end{array}$ & 0.665 & $0.483-0.915$ & 0.0122 \\
\hline Lymphocyte count & 1.00 & $0.999-1.00$ & 0.321 \\
\hline Hemoglobin & 0.789 & $0.621-1.00$ & 0.0511 \\
\hline Albumin & 0.180 & $0.0694-0.465$ & 0.000408 \\
\hline Lactate dehydrogenase & 1.00 & $0.998-1.00$ & 0.455 \\
\hline Calcium & 1.15 & $0.764-1.72$ & 0.51 \\
\hline C-reactive protein & 1.08 & $0.961-1.21$ & 0.196 \\
\hline \multicolumn{4}{|l|}{ Multivariate analysis } \\
\hline Bone metastasis & 1.98 & $0.666-5.90$ & 0.2190 \\
\hline Adrenal gland metastasis & 2.19 & $0.470-10.17$ & 0.3180 \\
\hline $\begin{array}{l}\text { Number of treatment } \\
\text { cycles }\end{array}$ & 0.744 & $0.518-1.07$ & 0.110 \\
\hline Albumin & 0.18 & $0.0638-0.508$ & 0.00121 \\
\hline
\end{tabular}

In the univariate analysis, serum albumin level, number of cycles, the existence of bone metastasis, and the existence of adrenal gland metastasis, all with $p$-values $<0.05$, were selected as candidate factors. A multivariate analysis identified serum albumin level as an independent factor associated with survival

Abbreviations: ECOG Eastern Cooperative Oncology Group

trials did not show statistically significant differences in OS [10-12]. In a real-world setting, patients were more heterogeneous and the proportion of frail patients was higher than those in clinical trials, thus the results of IFCT-0501 cannot apply entirely to the elderly population, especially patients with poor PS. The present study also revealed that the OS was significantly shorter in the early termination group than that in the continuous treatment group. Thus, for elderly patients with poor PS, consideration should be given to reasonably choose single-agent therapy, with low toxicity and continuation of as many cycles as possible. 
A serum albumin $\geq 3.4 \mathrm{~g} / \mathrm{dl}$

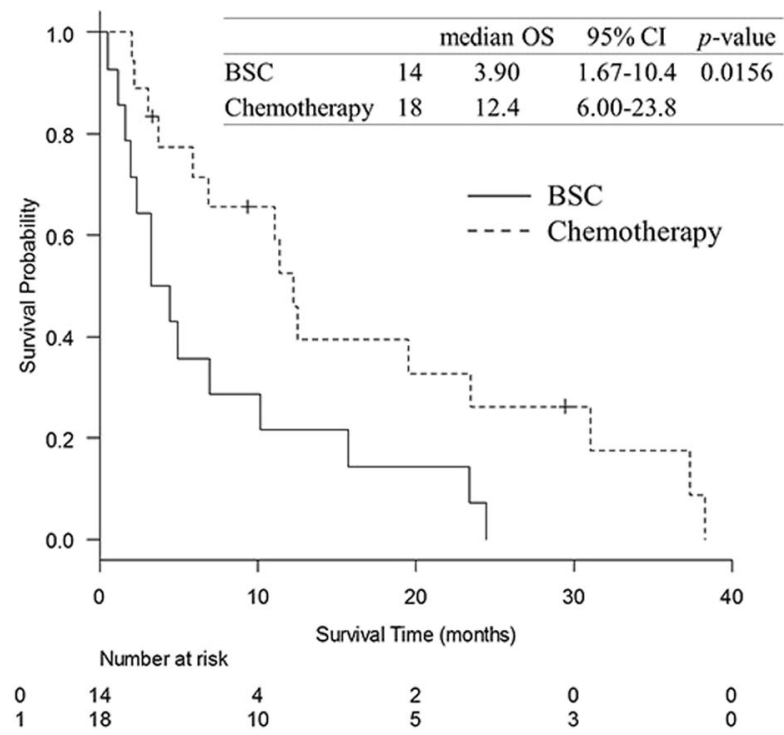

B serum albumin $<3.4 \mathrm{~g} / \mathrm{dl}$

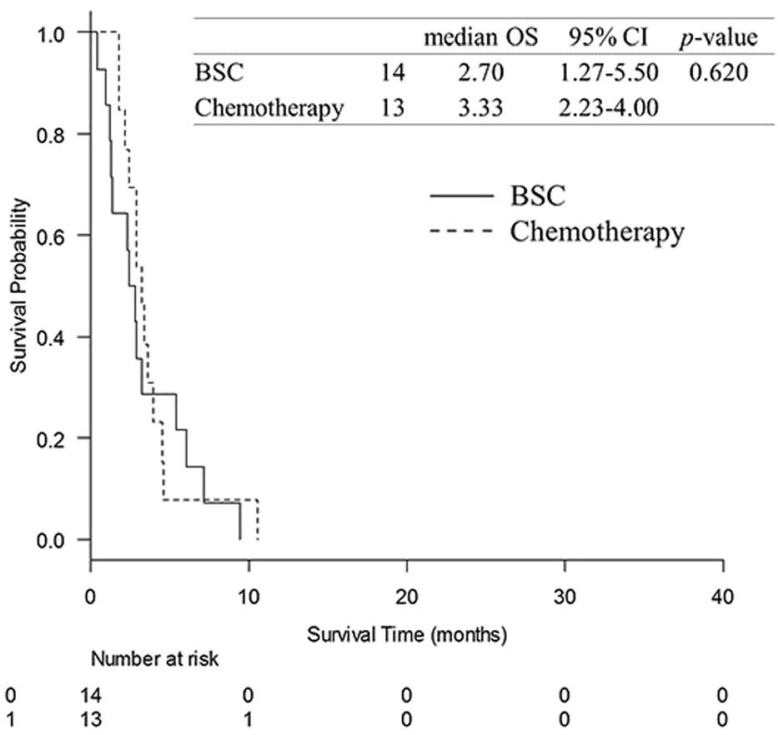

Fig. 3 Comparison of survival curves based on serum albumin levels. In the patients with serum albumin levels $\geq 3.40 \mathrm{~g} / \mathrm{dL}$, overall survival (OS) was significantly better in the chemotherapy group than that in the BSC group (a); in patients with serum albumin levels $<3.40 \mathrm{~g} / \mathrm{dL}$, the OS did not differ between chemotherapy and BSC groups (b)

For the treatment decision-making in elderly patients, geriatric assessment, including physical function, comorbidities, psychological state, social support, cognitive function, nutrition, and polypharmacy, is needed in conjunction with PS. Comprehensive geriatric assessment (CGA) has been adopted to evaluate elderly patients with cancer and may help identify patients who are fit and more likely to benefit from chemotherapy [13]. However, the recent ESOGIAGFPC-GECP 08-02 trial in elderly patients with advanced NSCLC failed to show a survival benefit of CGA-based strategy in spite of significantly fewer treatment failures attributed to toxicity [14]. In the present study, hypoalbuminemia was significantly associated with early termination of chemotherapy, and the patients without hypoalbuminemia received a significant survival benefit from chemotherapy. As one of the factors contributing to early termination, hypoalbuminemia was reported to correlate with grade $\geq 3$ non-hematological toxicity in elderly NSCLC patients [15]. On the other hand, the present study revealed that hypoalbuminemia was independently associated with survival in the chemotherapy group, and patients with hypoalbuminemia exhibited poor prognosis regardless of presence or absence of chemotherapy. Previous epidemiological works dissecting the association between pretreatment serum albumin levels and survival in NSCLC revealed that higher serum albumin levels were associated with better survival [16-23]. From these results, it was speculated that serum albumin level predicts the survival benefit of chemotherapy in elderly, advanced NSCLC patients with poor PS. In the CGA measurement tools, body mass index was often used for the assessment of nutrition status, whereas the serum albumin level was rarely used. The assessment tool including the serum albumin level, such as the Chemotherapy Risk Assessment Scale for High age (CRASH) score [15], may help identify patients more likely to benefit from chemotherapy.

A limitation of the present study was the retrospective single-center study design. Additionally, the number of included patients was small and the distribution of patients may have been skewed. There is a need to accumulate more cases from a plurality of hospitals and conduct further investigations for the validation of the present results. Factors associated with geriatric assessment, such as psychological state, social support, and cognitive function, were not fully evaluated. We might have to consider that prolongation of $\mathrm{OS}$ as an optimal endpoint for elderly, advanced NSCLC patients with poor PS.

\section{Conclusions}

In elderly, advanced NSCLC patients with poor PS, serum albumin levels may help identify certain populations more likely to receive a survival benefit of systemic chemotherapy.

\section{Additional file}

Additional file 1: Table S1. Comparison of clinical and laboratory data between the early termination group and the continuous treatment group (DOCX $14 \mathrm{~kb}$ ) 


\section{Abbreviations}

BSC: best supportive care; CGA: Comprehensive geriatric assessment; CRASH: Chemotherapy risk assessment scale for high age; ECOG: Eastern Cooperative Oncology Group; ELVIS: Elderly lung cancer vinorelbine italian study; MILES: Multicenter Italian Lung Cancer in the Elderly Study; NSCLC: non-small cell lung cancer; OS: overall survival; PFS: progression-free survival; PS: performance status; ROC: receiver operating characteristic

\section{Acknowledgements}

The authors would like to thank Morihito Takita and Atsuko Yoshizawa (Center for Advancing Translational Sciences, Kanagawa Prefectural Hospital Organization, Japan) for their advice concerning the statistical analysis.

\section{Funding}

This research received no specific grant from any funding agency in the public, commercial, or not-for-profit sectors.

\section{Availability of data and materials}

The datasets generated during and/or analyzed during the current study are available from the corresponding author on reasonable request.

\section{Authors' contributions}

Slked, $\mathrm{HY}$, and TI were involved in study concepts and design. SI (corresponding author), HY, Slkeo, MM, NS, TN, AN, and TY were involved in data acquisition; SIkeda and HY were involved in the quality control of data and algorithms; Slkeda, HY, SIkeo, MM, NS, TN, AN, TY, AS, TO, and TI were involved in the analysis and interpretation of the clinical data; Slkeda was involved in the statistical analysis; and SIkeda, HY, SIkeo, AS, TO, and TI were involved in drafting the manuscript. All authors read and approved the final manuscript.

\section{Ethics approval and consent to participate}

This study has been carried out in accordance with the Declaration of Helsinki. The Ethics Committee of the Kurashiki Central Hospital approved the study protocol. The Ethics Committee of the Kurashiki Central Hospital waived patient consent because this was a retrospective study and anonymity was secured.

\section{Consent for publication}

Not applicable.

\section{Competing interests}

S Ikeda, H Yoshioka, S Ikeo, M Morita, N Sone, T Niwa, A Nishiyama, T Yokoyama, A Sekine, T Ogura, and T Ishida declare that no potential conflicts of interest exist with any companies/organizations whose products or services may be discussed in this article.

\section{Publisher's Note}

Springer Nature remains neutral with regard to jurisdictional claims in published maps and institutional affiliations.

Received: 10 August 2017 Accepted: 21 November 2017

Published online: 28 November 2017

\section{References}

1. Altekruse S, Kosary S, Krapcho M, et al: SEER cancer statistics review, 19752007. http://seer.cancer.gov/archive/csr/1975_2007/

2. Lilenbaum RC, Cashy J, Hensing TA, et al. Prevalence of poor performance status in lung cancer patients: implications for research. J Thorac Oncol. 2008:3(2):125.

3. Iderly Lung Cancer Vinorelbine Italian Study Group. Effects of vinorelbine on quality of life and survival of elderly patients with advanced non-small cell lung cancer. J Natl Cancer Inst. 1999;91:66-72.

4. Gridelli C. The ELVIS trial: a phase III study of single-agent vinorelbine as firstline treatment in elderly patients with advanced non-small cell lung cancer Elderly lung cancer Vinorelbine Italian study. Oncologist. 2001;6(Suppl 1):4-7.

5. Gridelli C, Perrone F, Gallo C, et al. Chemotherapy for elderly patients with advanced non-small-cell lung cancer: the multicenter Italian lung cancer in the elderly study (MILES) phase III randomized trial. J Natl Cancer Inst. 2003; 95(5):362-72.

6. Kudoh S, Takeda K, Nakagawa K, et al. Phase III study of docetaxel compared with vinorelbine in elderly patients with advanced non-small cell lung cancer: results of the West Japan thoracic oncology group trial (WJTOG 9904). J Clin Oncol. 2006:24:3657-63.

7. Quoix E, Zalcman G, Oster J-P. Carboplatin and weekly paclitaxel doublet chemotherapy compared with monotherapy in elderly patients with advanced non-small-cell lung cancer: IFCT-0501 randomized, phase 3 trial. Lancet. 2011;378:1079-88.

8. Goldberg RM, Tabah-Fisch I, Bleiberg H, et al. Pooled analysis of safety and efficacy of oxaliplatin plus fluorouracil/leucovorin administered bimonthly in elderly patients with colorectal cancer. J Clin Oncol. 2006;24(25):4085-91.

9. Non-Small Cell Lung Cancer Collaborative Group. Chemotherapy and supportive care versus supportive care alone for advanced non-small cell lung cancer. Cochrane Database Syst Rev. 2010;5:CD007309.

10. Abe T, Takeda K, Ohe Y, et al. Randomized phase III trial comparing weekly docetaxel plus cisplatin versus docetaxel monotherapy every 3 weeks in elderly patients with advanced non-small-cell lung cancer: the intergroup trial JCOG0803/WJOG4307L. J Clin Oncol. 2015;33(6):575-81.

11. Chen YM, Perng RP, Shih JF, et al. A phase II randomized study of vinorelbine alone or with cisplatin against chemo-naïve inoperable nonsmall cell lung cancer in the elderly. Lung Cancer. 2008;61(2):214-9.

12. Lou GY, Li T, Gu CP, et al. Efficacy study of single-agent gemcitabine versus gemcitabine plus carboplatin in untreated elderly patients with stage IIIb/IV non-small-cell lung cancer. Zhonghua Yi Xue Za Zhi. 2010 Jan 12;90(2):100102 [article in Chinese].

13. Girre V, Falcou MC, Gisselbrecht M, et al. Does a geriatric oncology consultation modify the cancer treatment plan for elderly patients? J Gerontol A Biol Sci Med Sci. 2008;63:724-30.

14. Corre R, Greillier L, Le Caër $H$, et al. Use of a comprehensive geriatric assessment for the Management of Elderly Patients with Advanced nonSmall-Cell Lung Cancer: the phase III randomized ESOGIA-GFPC-GECP 08-02 study. J Clin Oncol. 2016;34(13):1476-83.

15. Extermann M, Boler I, Reich RR, et al. Predicting the risk of chemotherapy toxicity in older patients: the chemotherapy risk assessment scale for highage patients (CRASH) score. Cancer. 2012:118(13):3377-86.

16. Gupta D, Lis CG. Pretreatment serum albumin as a predictor of cancer survival: a systematic review of the epidemiological literature. Nutr J. 2010;9:69.

17. Win T, Sharples L, Groves AM, et al. Predicting survival in potentially curable lung cancer patients. Lung. 2008;186:97-102.

18. Forrest LM, McMillan DC, MCArdle CS, et al. A prospective longitudinal study of performance status, an inflammation-based score (GPS) and survival in patients with inoperable non-small-cell lung cancer. Br J Cancer. 2005;92:1834-6.

19. Lai SL, Perng RP. Impact of nutritional status on the survival of lung cancer patients. Zhonghua Yi Xue Za Zhi (Taipei). 1998:61:134-40.

20. Muers MF, Shevlin P, Brown J. Prognosis in lung cancer: physicians' opinions compared with outcome and a predictive model. Thorax. 1996;51:894-902.

21. Hespanhol V, Queiroga $H$, Magalhaes A, et al. Survival predictors in advanced non-small cell lung cancer. Lung Cancer. 1995:13:253-67.

22. Espinosa E, Feliu J, Zamora $P$, et al. Serum albumin and other prognostic factors related to response and survival in patients with advanced nonsmall cell lung cancer. Lung Cancer. 1995;12:67-76.

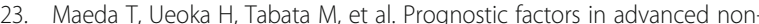
small cell lung cancer: elevated serum levels of neuron specific enolase indicate poor prognosis. Jpn J Clin Oncol. 2000;30:534-41.

\section{Submit your next manuscript to BioMed Central and we will help you at every step:}

- We accept pre-submission inquiries

- Our selector tool helps you to find the most relevant journal

- We provide round the clock customer support

- Convenient online submission

- Thorough peer review

- Inclusion in PubMed and all major indexing services

- Maximum visibility for your research

Submit your manuscript at www.biomedcentral.com/submit
BioMed Central 